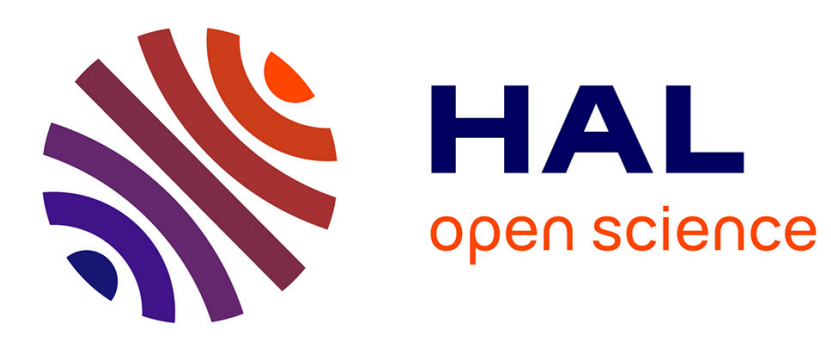

\title{
Sheared Amorphous Packings Display Two Separate Particle Transport Mechanisms
}

Dong Wang, Joshua A Dijksman, Jonathan Barés, Jie Ren, Hu Zheng

\section{To cite this version:}

Dong Wang, Joshua A Dijksman, Jonathan Barés, Jie Ren, Hu Zheng. Sheared Amorphous Packings Display Two Separate Particle Transport Mechanisms. Physical Review Letters, 2020, 125, pp.138001. 10.1103/PhysRevLett.125.138001 . hal-02953344

\section{HAL Id: hal-02953344 \\ https://hal.science/hal-02953344}

Submitted on 30 Sep 2020

HAL is a multi-disciplinary open access archive for the deposit and dissemination of scientific research documents, whether they are published or not. The documents may come from teaching and research institutions in France or abroad, or from public or private research centers.
L'archive ouverte pluridisciplinaire HAL, est destinée au dépôt et à la diffusion de documents scientifiques de niveau recherche, publiés ou non, émanant des établissements d'enseignement et de recherche français ou étrangers, des laboratoires publics ou privés. 


\title{
Sheared Amorphous Packings Display Two Separate Particle Transport Mechanisms
}

\author{
Dong Wang $\odot,{ }^{1, *}$ Joshua A. Dijksman $\odot,{ }^{2, *}$ Jonathan Barés, ${ }^{3}$ Jie Ren $\odot,{ }^{4}$ and Hu Zheng $\oplus^{1,5, \dagger}$ \\ ${ }^{1}$ Department of Physics and Center for Non-linear and Complex Systems, Duke University, Durham, North Carolina 27708, USA \\ ${ }^{2}$ Physical Chemistry and Soft Matter, Wageningen University and Research, Stippeneng 4, 6708 WE Wageningen, Netherlands \\ ${ }^{3}$ LMGC, UMR 5508 CNRS-University Montpellier, 34095 Montpellier, France \\ ${ }^{4}$ Merck \& Company, Incorporated, West Point, Pennsylvania 19486, USA \\ ${ }^{5}$ Department of Geotechnical Engineering, College of Civil Engineering, Tongji University, Shanghai 200092, China
}

(Received 23 December 2019; revised 12 July 2020; accepted 9 September 2020; published 24 September 2020)

\begin{abstract}
Shearing granular materials induces nonaffine displacements. Such nonaffine displacements have been studied extensively, and are known to correlate with plasticity and other mechanical features of amorphous packings. A well known example is shear transformation zones as captured by the local deviation from affine deformation, $D_{\min }^{2}$, and their relevance to failure and stress fluctuations. We analyze sheared frictional athermal disc packings and show that there exists at least one additional mesoscopic transport mechanism that superimposes itself on top of local diffusive motion. We evidence this second transport mechanism in a homogeneous system via a diffusion tensor analysis and show that the trace of the diffusion tensor equals the classic $D_{\min }^{2}$ when this second mesoscopic transport is corrected for. The new transport mechanism is consistently observed over a wide range of volume fractions and even for particles with different friction coefficients and is consistently observed also upon shear reversal, hinting at its relevance for memory effects.
\end{abstract}

DOI: 10.1103/PhysRevLett.125.138001

Sheared disordered materials have peculiar mechanical properties, including the tendency to either dilate or contract with strain [1-7], display peak stress behavior [8] and remember the way they were mechanically excited in their past [9-12]. These mechanical nontrivialities are most pronounced when a packing of particles is almost but not entirely rigid, as set by the particle volume fraction. Around this volume fraction, shear has the ability to "jam" or rigidify disordered packings $[2,13]$. The underpinnings of the mechanism are still under intense debate [14-19]. One of the complicating factors is that many of the nontrivial mechanical features are transient phenomena, making them particularly difficult to probe, as they are highly dependent on packing preparation, boundary conditions, and other seemingly insignificant details of the system [7,15]. In the past, qualitative and quantitative features such as shear transformation zones [20-23], dynamical heterogeneities [24-26], anisotropy [27-29], and vortices [30] have all been observed in the transient response of amorphous packings exposed to shear. However, it is not clear which of these transport phenomena are relevant for the observed mechanics, or how many of these occur simultaneously during shear.

We show here that particle displacements during the startup transient in a sheared amorphous packing is dominated by two simultaneous but distinct particle transport mechanisms, only one of which is sensitive to the particle volume fraction. Besides the locally nonaffine, diffusive particle motion as quantified by the standard measurement of the local deviation from affine deformation $D_{\min }^{2}$ as defined in Refs. [20,21], we observe a directional, collective particle transport mechanism that occurs on a length scale of up to ten times the shear transformation zone size. This directional transport, from now on defined as $D^{1}$, occurs before the emergence of dilatancy-induced Reynolds pressure yet disappears in the random close packing limit. Moreover, the onset of $D^{1}$ emerges at larger $\phi$ for lower particle friction coefficients, which hints at its relevance for shear jamming phenomena, which display similar trends $[3,13]$. Interestingly, $D^{1}$ survives cyclic shear over many different cycles, converges to limit cycle behavior and displays strain dependence in its response to cyclic driving, making $D^{1}$ of interest as metric to capture the dynamics of memory formation in amorphous packings $[7,12,15,31-36]$.

To observe particle transport in disordered media, we use a setup introduced before $[3,18]$. We apply simple shear to bidisperse granular systems composed of photoelastic discs (diameter ratio $1: 1.25$ ) with different interparticle friction coefficients, $\mu$. Shear is performed in an apparatus that suppresses shear bands and related shear-induced density fluctuations entirely [3]. Particles were carried by the separate slats that form the shear box base. These slats move affinely in accordance with the applied shear (see more details in Supplemental Material [37]). The optical properties of photoelastic discs reveals particle-scale contact forces when placed between a pair of crossed polarizers [42-44]. Discs were cut from photoelastic sheets 
(Vishay PSM-4), resulting in a $\mu \approx 0.7[3,44]$. Different $\mu$ were achieved by either wrapping these particles with Teflon tape, reducing $\mu$ to $\approx 0.15$, or making another set of photoelastic discs with fine teeth on the edge [44]. For a picture of the particles used, see the Supplemental Material [37]. Henceforth, we will refer to particles with $\mu \approx 0.15$, $\mu \approx 0.7$, and fine teeth as $\mu=\mu_{l}, \mu=\mu_{m}$, and $\mu=\mu_{h}$ particles, respectively. The system contained approximately $45 \times 20$ discs with a large to small number ratio 1:3.3 to prevent crystallization. Every run at given packing fraction $\phi$ was repeated five times; the initial stress-free state was prepared anew for each run. For every friction coefficient, we explored a range of $\phi$ in which we could observe the emergence of dilatancy pressure within the strain amplitude achievable by the apparatus.

Shear was applied quasi-statically in the $y$ direction to a shear box [Fig. 1(a)]. Starting from a stress-free state, the system was sheared by strain step of $\delta \gamma=0.0027$. Then the system was left to relax for six seconds, followed by taking two images, which reveal information on particle position and photoelastic response. Such a process-stepwise
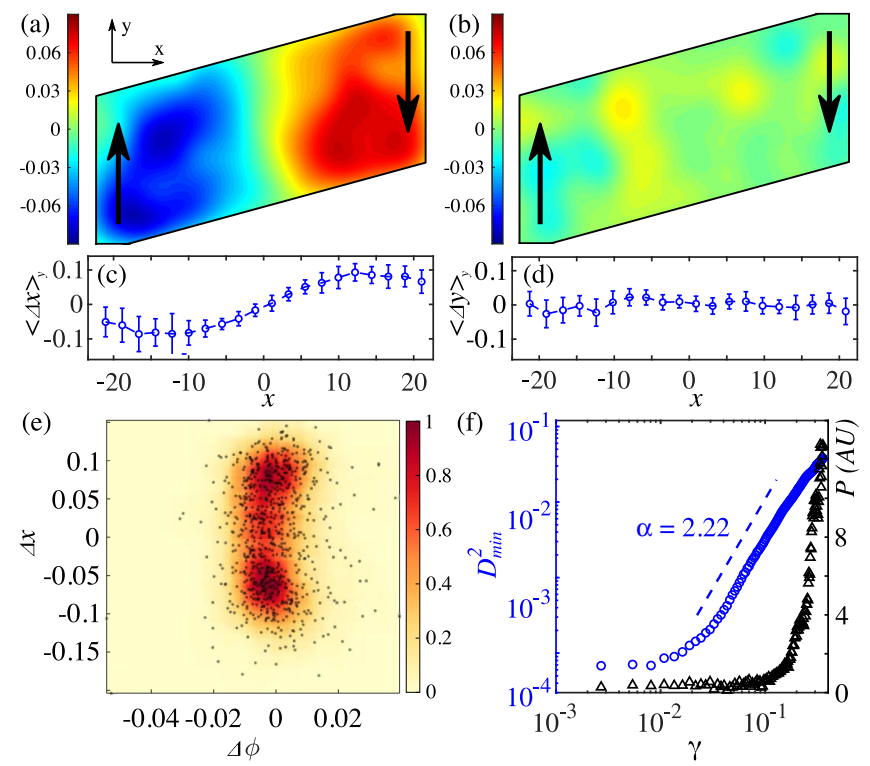

FIG. 1. Example of the system response to simple shear at packing fraction 0.78. (a),(b) Coarse grained nonaffine $x$ and $y$ displacements after a shear strain $\gamma=0.054$. The Cartesian coordinates are given in (a). The black arrows indicate the applied shear direction. (c),(d) Nonaffine $x$ and $y$ displacements averaged along the $y$ axis, as a function of $x$. Data are from the same states as (a) and (b), respectively. The dashed lines are a guide to the eye. $x=0$ is at the center of the shear box. (e) Particles' nonaffine $x$ displacements as a function of their local packing fraction change for each particle. The colored mask shows the normalized probability distribution of the points with the probability indicated by the color bar. (f) Local deviation from the affine deformation $D_{\min }^{2}$ (left, blue circles) and system pressure (right, black triangles) as a function of shear strain $\gamma$. All lengths are in the unit of average particle diameter $\bar{D}$. shearing, relaxing and imaging-was repeated until a certain amount of total strain was achieved. The maximum shear strain $\gamma$ that can be achieved here is 0.54 , which is the limit of the experimental setup. From the images, we tracked particle positions and computed particle-scale and system-wide pressure by averaging light intensity gradient squared [42-44]. Henceforth, we use the average diameter of particles in the system $\bar{D}$ as the unit length in all results.

Two transport mechanisms can be qualitatively observed under all experimental conditions. We can quantify the transport by decomposing the displacement field into the $x$ and $y$ component, which is referred to as the shear and perpendicular directions respectively [see Fig. 1(a)]. We compute particle displacements at any given $\gamma$ compared to $\gamma=0$. Figures 1(a) and 1(b) show typical results of particle nonaffine $x$ and $y$ displacements at $\phi=0.78$ and $\gamma=0.054$, coarse grained by a Gaussian function with a half width of $2 \bar{D}[45,46]$. In the $x$ direction, the expected affine displacement field is zero, but we see a substantial amount of particle displacement away from the center of the box. For the $y$ direction, the expected global affine motion profile is linear; this have already been verified [3]. Subtracting the globally affine linear motion reveals that on top of the linear deformation profile, a small perturbation is visible in Fig 1(b); however, the perturbation is spatially more heterogeneous and much smaller than the transport in the $x$ direction, as evidenced by the mean transport $\langle\Delta x\rangle_{y}(x),\langle\Delta y\rangle_{y}(x)$ across the system that are averaged over $y$ direction, as shown in Figs. 1(c) and 1(d). Hence, from now on, we only look at the binned $x$-dependent displacement data, i.e., $\langle\Delta x\rangle_{y}(x)$ and $\langle\Delta y\rangle_{y}(x)$.

We can see that the displacements induced by the shear are not eased by local free volume availability. $\langle\Delta x\rangle_{y}$ is clearly a function of $x$, and one might relate this dependence with the local deviations $\Delta \phi$ from the mean density $\phi$. However, we see no correlation between $\Delta x$ and $\Delta \phi$ when we plot them together as shown in Fig. 1(e). Local nonaffine displacements should be induced by local pressure fluctuations [47], yet as shown by Fig. 1(f) we find that the total nonaffine motion induced by shear is not sensitive to the average global pressure in the system. In Fig. 1(f), we quantify the overall nonaffine displacement with the standard $D_{\min }^{2}$ metric and see that it rises independently of the evolving Reynolds pressure in the system. Here $D_{\text {min }}^{2}$ is calculated by averaging over all the particles the following quantity, as done previously $[20,21]$ :

$$
D_{\min , \mathrm{i}}^{2}(\gamma)=\frac{1}{N_{i}} \min _{\mathbf{J}_{i}} \sum_{j \in N_{i}}\left[\mathbf{r}_{i j}(\gamma)-\mathbf{J}_{i} \mathbf{r}_{i j}(\gamma=0)\right]^{2},
$$

where $j$ is the index of all $N_{i}$ particles that are within $2 \bar{D}$ distance to particle $i$ center, $\mathbf{r}_{i j}(\gamma)$ is the vector from particle $i$ to particle $j$ at a given strain $\gamma$, and $\mathbf{J}_{i}$ is the fitted strain field that minimizes the above quantity. 


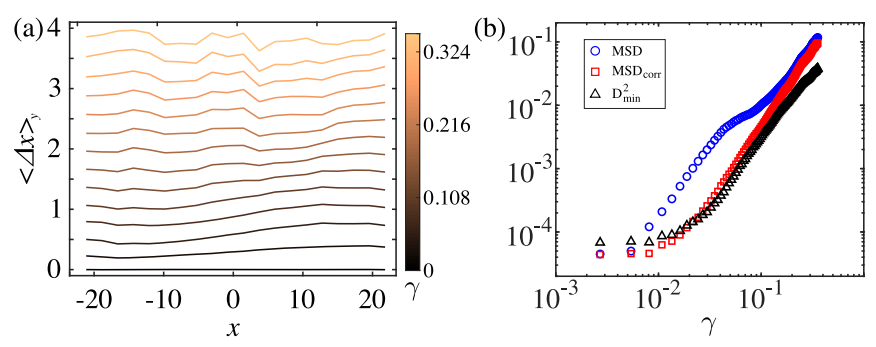

FIG. 2. (a) Nonaffine $x$ displacements $\Delta x$, averaged in the same way as Fig. 1(c), as a function of the bin position for different shear strains $\gamma$. Different $\gamma \mathrm{s}$ are indicated by the colorbar and the curves are shifted for better visibility. (b) Mean squared displacement (MSD) before (triangles) and after (circles) correcting the diffusion tensor $T$ with $\langle\Delta x\rangle_{y}$ and $\langle\Delta y\rangle_{y}$ from the nonaffine displacements, and $D_{\min }^{2}$ (squares), vs $\gamma$. Data from the same run in Fig. 1. All lengths are in the unit of average particle diameter $\bar{D}$.

In Figs. 2(b) and 1(f), we observe that $D_{\min }^{2}=\left\langle D_{\min , \mathrm{i}}^{2}\right\rangle_{i}$ dynamics in the packings is entirely consistent with earlier work on a completely different system [21], even though we use here a constant volume setting and observe an additional mesoscopic transport mechanism (see more details in the Supplemental Material [37]). We conclude that $D_{\min }^{2}$ is a local background process that is largely insensitive to the details of the deformations imposed.

Identifying two different mechanisms.-We extract the additional mesoscopic transport mechanisms that occur simultaneously with $D_{\min }^{2}$ by measuring $\langle\Delta x(x, \gamma)\rangle_{y}$; see Fig. 2(a). We observe that the directional displacement emerges immediately at small $\gamma$ but later becomes more spatially heterogeneous over smaller length scales (see details in the Supplemental Material [37]). To further show that the directional transport $D^{1}$ is independent of locally nonaffine "random" motion, we compute the diffusion tensor of the particle displacement

$$
T=\left\langle\left(\begin{array}{cc}
\Delta x^{2} & \Delta x \cdot \Delta y \\
\Delta y \cdot \Delta x & \Delta y^{2}
\end{array}\right)\right\rangle,
$$

where $\langle\cdots\rangle$ is the average over all particles. We compute this tensor in two ways: once before and once after subtracting the local mesoscale displacement fluctuations $\langle\Delta x\rangle_{y},\langle\Delta y\rangle_{y}$ from the displacement of the particles. From these two tensors we compute the trace and call them MSD and $\mathrm{MSD}_{\text {corr }}$, respectively. We note that subtracting only $D^{1}$ and not $\langle\Delta y\rangle_{y}$ gives similar results and the symmetric offdiagonal term is nonzero, which indicates that the major diffusion directions are not along $x$ or $y$ axes (see the Supplemental Material [37]). The result in Fig. 2(b) indicates that after subtracting the mesoscale displacements, the trace of the diffusion tensor coincides with the $D_{\min }^{2}$ metric: without mesoscale displacements, nonaffine motion cannot be distinguished from diffusive behavior, consistent with earlier results [21].
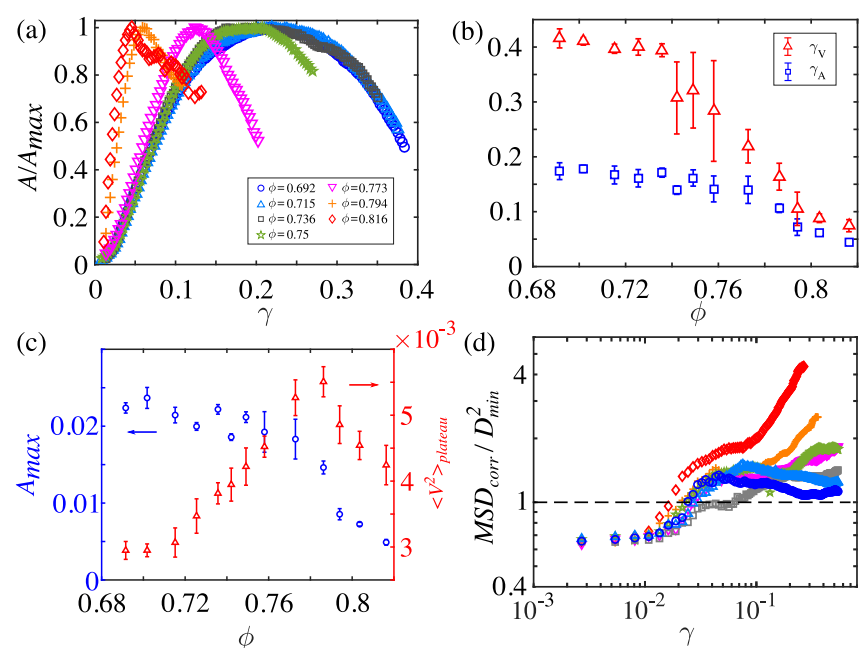

FIG. 3. (a) Linear slope $A$ extrapolated from $\langle\Delta x\rangle_{y}$ vs $x$ for $x$ in the range $(-10,10)$, normalized by its maximum value in a run $A_{\max }$, vs shear strain $\gamma$, at different $\phi$ as indicated by different symbols and colors. (b) Corresponding $\gamma$ at $A_{\max }$ (left, circles), and $\gamma_{\text {onset }}$ for average vorticity $\left\langle V^{2}\right\rangle$ to reach the plateau (right, triangles), and (c) $A_{\max }$ (left, circles), and plateau value of $\left\langle V^{2}\right\rangle$ (right, triangles), vs $\phi$, vs $\phi$, averaged over five runs for each $\phi$. Arrows indicate axes associated with the data. (d) The ratio between $\mathrm{MSD}_{\text {corr }}$ and $D_{\min }^{2}$ vs $\gamma$, at various packing fractions $\phi$ as indicated the same way in (a). The dashed line corresponds to the ratio being 1 . All lengths are in the unit of average particle diameter $\bar{D}$.

One expects the strain needed to induce particle transport to decrease with $\phi$; this is indeed the case. Figure 3(a) shows the amplitude of $D^{1}$ as measured by $A=$ $d\langle\Delta x\rangle_{y} / d x \mid(x=0)$ as a function of strain, measuring the spatial gradient of the orthogonal displacement field in the center of the shear box. $A$ peaks at $A_{\max }$, and this peak is reached at progressively smaller strain amplitudes (denoted as $\gamma_{A}$ ) as $\phi$ increases, as can be seen in Fig. 3(b). However, the amplitude of $D^{1}$ is largest at small $\phi$. As shown in Fig. 3(c), $A_{\max }$ drops with increasing $\phi$ and seems to disappear around the random close packing limit of $\sim 0.83$, where also the strain needed to reach peak $D^{1}$ vanishes. Our data indicates that the $D^{1}$ only disappears at packing fractions at which it is no longer possible to create stress free initial configurations [48]. This suggests that $D^{1}$ no longer exists when the system approaches the density at which no or little shear is required to induce a finite pressure, signifying that all particles are sterically hindered. Note that $D^{1}$ changes character already at packing fractions $(\sim 0.74)$ far below the random close packing limit, which coincides with the lowest $\phi$ needed for shear induced jamming to occur $[3,13]$. We can see this by probing the density dependent difference between $\mathrm{MSD}_{\text {corr }}$ and $D_{\min }^{2}$, as shown in Fig. 3(d). For low $\phi$ we observe that these two local displacement metrics essentially coincide over the entire range of strains, whereas for larger $\phi$ we see a monotonic growth of their difference. This stems from the 
fact that the $D^{1}$ field gets more and more directionally heterogeneous at large $\gamma$ as the volume fraction increases towards random close packing. Note that $\mathrm{MSD}_{\text {corr }} / D_{\min }^{2}$ is almost $\phi$ independent for $\gamma$ smaller than 0.01; we attribute that to the plateau of $\mathrm{MSD}_{\text {corr }}$ and $D_{\min }^{2}$, which is most likely caused by tracking precision (see the Supplemental Material [37]), as shown in Fig. 2(b).

To further test the uniqueness of the $D^{1}$ transport, we compute the vorticity $V$ of the nonaffine grain flow (see [37]). We see that the value of $V^{2}$ averaged over the system, $\left\langle V^{2}\right\rangle$, first grows steadily with $\gamma$ and then reaches a plateau (see [37]). The strain at which $\left\langle V^{2}\right\rangle$ reaches the plateau, $\gamma_{V}$, shown in Fig. 3(b) in comparison with $\gamma_{A}$, indicates that vortices develop and exist at a much longer time or strain scale than $D^{1}$ transport. This observation manifests the importance of $D^{1}$ transport: vorticity cannot capture $D^{1}$. The plateau value of $\left\langle V^{2}\right\rangle,\left\langle V^{2}\right\rangle_{\text {plateau }}$, first increases and then decreases as $\phi$ increases [see Fig. 3(c)]. The change in trend occurs around $\sim 0.79$, which appears to be the point where $\gamma_{A}$ and $\gamma_{V}$ start to behave differently. Note that observing a trend change at this point seems to be consistent with results reported in works on glass transition in granular systems $[49,50]$ and the isotropic jamming point in frictional systems under compression $[19,38]$.

To highlight the subtle role of $D^{1}$ for amorphous packing dynamics, we probe how this transport mechanism depends on the initial boundary conditions (IBC) and responds to cyclic shear. As shown in Fig. 4(a), initiating shear from a rectangular box instead of a parallelogram inverts the direction of $D^{1}$. We show additional data in the Supplemental Material [37] that other transport mechanisms than $D^{1}$ are not affected by IBC. This sensitivity to IBC makes $D^{1}$ a prime candidate for elucidating the emergence of memory in granular packings [7,34-36]. While an in-depth study of $D^{1}$ in memory formation is left for future work, we can see in Fig. 4(a) that the $D^{1}$ amplitude shows a distinct change in behavior beyond a critical strain amplitude, and note that memory formation in

(a)
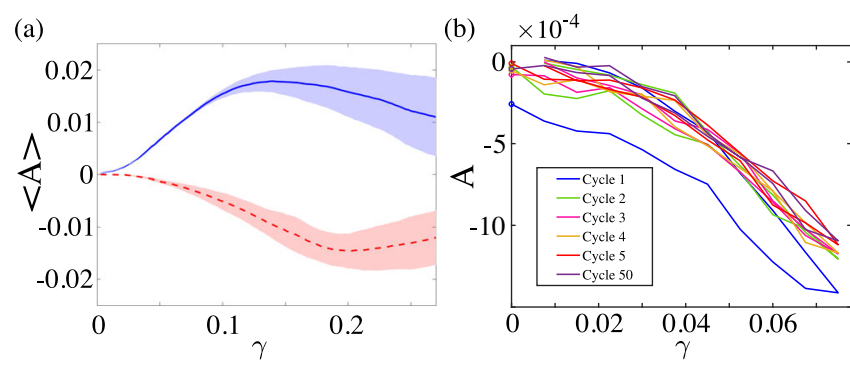

FIG. 4. (a) Examples of $D^{1}$ amplitude $A$ vs shear strain $\gamma$ for two different initial shear cell shapes at $\phi=0.78$, averaged over five runs: parallelogram (blue solid line) and rectangle (red dashed line). Shaded areas correspond to standard deviations. (b) $A$ vs $\gamma$ for a packing undergoing cyclic shear. Colors indicate different cycle number. Circles mark the end of corresponding cycles. sheared amorphous packings is also strongly strain dependent. Another hallmark of structural memory formation is also observed in $D^{1}$ transport in Fig. 4(b). Transport over repeated cycles below the critical strain amplitude induces a relaxation towards limit cycle behavior. In previous work on cyclic shear dynamics in the same system, relaxation dynamics towards cyclic dynamics was also observed in the pressure of the packing. However, the pressure dynamics evolved towards a strain-symmetric response [3]. The absence of such relaxation dynamics in the microstructural dynamics as captured by $D^{1}$, and the sign change in its directionality depending on the initial conditions of the shear is striking. We see here a surprising disconnect between microstructural and pressure dynamics in amorphous packings and these observations amplify the importance of probing microstructural dynamics in amorphous packings, for example by looking at $D^{1}$.

Discussion.-Our findings provide a new path to understanding the mechanisms of transport for sheared amorphous materials, especially at the transient stage where also shear jamming occurs $[2,18]$. Besides $D_{\min }^{2}$, which we show to be greatly insensitive to packing conditions, there are clearly other transport mechanisms emerging that affect the dynamics of sheared amorphous particle packings. The disappearance of $D^{1}$ transport at larger $\phi$ yet below the random close packing limit indicates that there might be a third transport mechanism that cannot be well characterized within our experimental resolution. This clearly suggests new perspectives for numerical work: it would be interesting to see if the $D^{1}$ dynamics as observed in our model system can also be observed in numerical simulations; as they appear in the transient behavior, obtaining statistics should be computationally cheap comparing to those in the steady state. In fact, $D^{1}$ metrics can be used to efficiently verify the performance of numerical simulations, as they appear immediately after shear is initiated. Another question is how to capture $D^{1}$ and other such transport processes in models. Note that theoretical considerations [27-29] had already suggested that anisotropic displacement fields are a necessary ingredient to understand the dynamics of sheared amorphous system; our experimental data supports such perspectives.

Conclusions.-We have studied particle transport in an amorphous particle packing and revealed that multiple distinct transport mechanisms occur at the same time. The experimental system in which we made these observations was composed of photoelastic discs exposed to quasistatic shear with various packing fractions $\phi$ and interparticle friction coefficients $\mu$. We paid particular attention to the transient stage and found that there exist two distinct nonaffine transport mechanisms in response to shear: ballistic random-walk-like diffusion that can be well characterized by $D_{\min }^{2}$, and a second collective directional displacement phenomenon, which we denote as $D^{1}$ transport. The $D_{\min }^{2}$ dynamics appears insensitive to either $\phi$ or 
$\mu$, while $D^{1}$ transport is sensitive to both. We find that when $D^{1}$ is subtracted from the displacement field, the trace of the diffusion tensor coincides with $D_{\min }^{2}$, further solidifying the distinct nature of these two mechanisms. In the range of packing fractions just below random close packing, the $D^{1}$ correction to the overall displacement field does not isolate the diffusive motion very well anymore, suggesting that either the $D^{1}$ transport changes in character, or additional displacement mechanisms become relevant.

We are very grateful to discussions with Corey S. O'Hern, Brian P. Tighe, Lou Kondic, Joshua E.S. Socolar, and Pierre Ronceray. We greatly thank the late Robert P. Behringer for his initial support and intellectual input to this work. This work was supported by NSFC Grant No. 41672256 (H.Z.), NSF Grants No. DMR1206351 and No. DMR1809762, and the W. M. Keck Foundation.

*These authors contributed equally. †henghu@tongji.edu.cn

[1] O. Reynolds, On the dilatancy of media composed of rigid particles in contact, with experimental illustrations, Phil. Mag. 20, 469 (1885).

[2] D. Bi, J. Zhang, B. Chakraborty, and R. P. Behringer, Jamming by shear, Nature (London) 480, 355 (2011).

[3] J. Ren, J. A. Dijksman, and R. P. Behringer, Reynolds Pressure and Relaxation in a Sheared Granular System, Phys. Rev. Lett. 110, 018302 (2013).

[4] O. Pouliquen, M. Belzons, and M. Nicolas, Fluctuating Particle Motion During Shear Induced Granular Compaction, Phys. Rev. Lett. 91, 014301 (2003).

[5] J.-C. Tsai, G. A. Voth, and J. P. Gollub, Internal Granular Dynamics, Shear-Induced Crystallization, and Compaction Steps, Phys. Rev. Lett. 91, 064301 (2003).

[6] A. J. Kabla and T. J. Senden, Dilatancy in Slow Granular Flows, Phys. Rev. Lett. 102, 228301 (2009).

[7] M. Toiya, J. Stambaugh, and W. Losert, Transient and Oscillatory Granular Shear Flow, Phys. Rev. Lett. 93, 088001 (2004).

[8] M. Oda, J. Konishi, and S. Nemat-Nasser, Experimental micromechanical evaluation of strength of granular materials: Effects of particle rolling, Mech. Mater. 1, 269 (1982).

[9] N. C. Keim, J. D. Paulsen, Z. Zeravcic, S. Sastry, and S. R. Nagel, Memory formation in matter, Rev. Mod. Phys. 91, 035002 (2019).

[10] L. Vanel, D. Howell, D. Clark, R. P. Behringer, and E. Clément, Memories in sand: Experimental tests of construction history on stress distributions under sandpiles, Phys. Rev. E 60, R5040 (1999).

[11] P. Umbanhowar and M. van Hecke, Force dynamics in weakly vibrated granular packings, Phys. Rev. E 72, 030301 (E) (2005).

[12] A. Peshkov, M. Girvan, D. C. Richardson, and W. Losert, Reversibility of granular rotations and translations, Phys. Rev. E 100, 042905 (2019).

[13] Y. Zhao, J. Barés, H. Zheng, J. E. S. Socolar, and R. P. Behringer, Shear-Jammed, Fragile, and Steady States in
Homogeneously Strained Granular Materials, Phys. Rev. Lett. 123, 158001 (2019).

[14] S. Sarkar and B. Chakraborty, Shear-induced rigidity in athermal materials: A unified statistical framework, Phys. Rev. E 91, 042201 (2015).

[15] N. Kumar and S. Luding, Memory of jamming - multiscale models for soft and granular matter, Granular Matter 18, 58 (2016).

[16] T. Bertrand, R. P. Behringer, B. Chakraborty, C. S. O’Hern, and M. D. Shattuck, Protocol dependence of the jamming transition, Phys. Rev. E 93, 012901 (2016).

[17] M. Baity-Jessi, C. P. Goodrich, A. J. Liu, S. R. Nagel, and J. P. Sethna, Emergent so(3) symmetry of the frictionless shear jamming transition, J. Stat. Phys. 167, 735 (2017).

[18] D. Wang, J. Ren, J. A. Dijksman, H. Zheng, and R. P. Behringer, Microscopic Origins of Shear Jamming for 2d Frictional Grains, Phys. Rev. Lett. 120, 208004 (2018).

[19] F. Xiong, P. Wang, A. H. Clark, T. Bertrand, N. T. Ouellette, M. D. Shattuck, and C. S. O'Hern, Comparison of shear and compression jammed packings of frictional disks, Granular Matter 21, 109 (2019).

[20] M. L. Falk and J. S. Langer, Dynamics of viscoplastic deformation in amorphous solids, Phys. Rev. E 57, 7192 (1998).

[21] W. Li, J. M. Rieser, A. J. Liu, D. J. Durian, and J. Li, Deformation-driven diffusion and plastic flow in amorphous granular pillars, Phys. Rev. E 91, 062212 (2015).

[22] G. Lois, A. Lemaître, and J. M. Carlson, Numerical tests of constitutive laws for dense granular flows, Phys. Rev. E 72, 051303 (2005)

[23] A. Le Bouil, A. Amon, S. McNamara, and J. Crassous, Emergence of Cooperativity in Plasticity of Soft Glassy Materials, Phys. Rev. Lett. 112, 246001 (2014).

[24] A. Mehta, G. C. Barker, and J. M. Luck, Heterogeneities in granular dynamics, Proc. Natl. Acad. Sci. U.S.A. 105, 8244 (2008).

[25] L. Berthier, G. Biroli, J.-P. Bouchaud, L. Cipelletti, and W. van Saarloos, Dynamical Heterogeneities in Glasses, Colloids, and Granular Media, Vol. 150 (Oxford University Press, Oxford, 2011).

[26] J. Barés, D. Wang, D. Wang, T. Bertrand, C. S. O'Hern, and R. P. Behringer, Local and global avalanches in a twodimensional sheared granular medium, Phys. Rev. E 96, 052902 (2017).

[27] R. N. Chacko, R. Mari, S. M. Fielding, and M. E. Cates, Shear reversal in dense suspensions: The challenge to fabric evolution models from simulation data, J. Fluid Mech. 847, 700 (2018).

[28] A. Misra and P. Poorsolhjouy, Granular micromechanics based micromorphic model predicts frequency band gaps, Continuum Mech. Thermodyn. 28, 215 (2016).

[29] P. Poorsolhjouy and A. Misra, Granular micromechanics based continuum model for grain rotations and grain rotation waves, J. Mech. Phys. Solids 129, 244 (2019).

[30] S. L. Conway, T. Shinbrot, and B. J. Glasser, A taylor vortex analogy in granular flows, Nature (London) 431, 433 (2004).

[31] F. Gadala-Maria and A. Acrivos, Shearinduced structure in a concentrated suspension of solid spheres, J. Rheol. 24, 799 (1980). 
[32] N. C. Keim and S. R. Nagel, Generic Transient Memory Formation in Disordered Systems with Noise, Phys. Rev. Lett. 107, 010603 (2011).

[33] J. D. Paulsen, N. C. Keim, and S. R. Nagel, Multiple Transient Memories in Experiments on Sheared NonBrownian Suspensions, Phys. Rev. Lett. 113, 068301 (2014).

[34] D. Fiocco, G. Foffi, and S. Sastry, Encoding of Memory in Sheared Amorphous Solids, Phys. Rev. Lett. 112, 025702 (2014).

[35] J. R. Royer and P. M. Chaikin, Precisely cyclic sand: Self-organization of periodically sheared frictional grains, Proc. Natl. Acad. Sci. U.S.A. 112, 49 (2015).

[36] B. Kou, Y. Cao, J. Li, C. Xia, Z. Li, H. Dong, A. Zhang, J. Zhang, W. Kob, and Y. Wang, Granular materials flow like complex fluids, Nature (London) 551, 360 (2017).

[37] See the Supplemental Material at http://link.aps.org/ supplemental/10.1103/PhysRevLett.125.138001 for a detailed description of the experimental setup, $\mathrm{MSD}_{\text {corr }}$ with only the $x$ directional transport correction, anisotropy in $T$, detailed $D^{1}$ transport amplitude calculation, more results on the deviatoric shear strain, detailed vorticity calculation, and dependence of diffusion amplitude on $\phi$ and $\mu$, which includes Refs. [3,19-21,38-41].

[38] L. E. Silbert, Jamming of frictional spheres and random loose packing, Soft Matter 6, 2918 (2010).

[39] M. Suzuki, T. Shinmura, K. Iimura, and M. Hirota, Study of the wall effect on particle packing structure using $\mathrm{x}$-ray micro computed tomography, Adv. Powder Technol. 19, 183 (2008).

[40] S. C. du Pont, P. Gondret, B. Perrin, and M. Rabaud, Wall effects on granular heap stability, Europhys. Lett. 61, 492 (2003).
[41] S. Luding, Granular matter: So much for the jamming point, Nat. Phys. 12, 531 (2016).

[42] D. Howell and R. P. Behringer, Fluctuations and dynamics for a two-dimensional sheared granular material, in Powders and Grains 97, edited by R. P. Behringer and J. T. Jenkins (Taylor \& Francis, London, 1997), pp. 337-340.

[43] D. Howell, R. P. Behringer, and C. Veje, Stress Fluctuations in a 2d Granular Couette Experiment: A Continuous Transition, Phys. Rev. Lett. 82, 5241 (1999).

[44] A. A. Zadeh, J. Barés, T. A. Brzinski, K. E. Daniels, J. A. Dijksman, N. Docquier, H. Everitt, J.E. Kollmer, O. Lantsoght, D. Wang, M. Workamp, Y. Zhao, and H. Zheng, Enlightening force chains: A review of photoelasticimetry in granular matter, Granular Matter 21 (2019).

[45] J. Zhang, R. P. Behringer, and I. Goldhirsch, Coarsegraining of a physical granular system, Prog. Theor. Phys. Suppl. 184, 16 (2010).

[46] A. H. Clark, P. Mort, and R. P. Behringer, Coarse graining for an impeller-driven mixer system, Granular Matter 14, 283 (2012).

[47] W. Losert, L. Bocquet, T. C. Lubensky, and J. P. Gollub, Particle Dynamics in Sheared Granular Matter, Phys. Rev. Lett. 85, 1428 (2000).

[48] T. S. Majmudar, M. Sperl, S. Luding, and R. P. Behringer, Jamming Transition in Granular Systems, Phys. Rev. Lett. 98, 058001 (2007).

[49] A. S. Keys, A. R. Abate, S. C. Glotzer, and D. J. Durian, Measurement of growing dynamical length scales and prediction of the jamming transition in a granular material, Nat. Phys. 3, 260 (2007).

[50] R. Candelier, O. Dauchot, and G. Biroli, Dynamical facilitation decreases when approaching the granular glass transition, Europhys. Lett. 92, 24003 (2010). 